\title{
Asymptotic behavior of parametric estimation for a class of nonlinear diffusion process
}

\author{
Chenglian Zhu \\ School of Mathematical Science, Huaiyin Normal University, Huaian, Jiangsu 223300, P. R. China. \\ Communicated by M. Bohner
}

\begin{abstract}
In this paper, a stochastic process, which is a class of nonhomogeneous diffusion process from the perspective of the corresponding nonlinear stochastic differential equation is studied. The parameter included in the drift term are estimated by sequential maximum likelihood methodology. The sequential estimators are proved to be closed, unbiased, strongly consistent, normally distributed, and optimal in the mean square sense.
\end{abstract}

Keywords: Nonlinear diffusion process, sequential maximum likelihood estimation, mean square sense. 2010 MSC: 60F15.

(C) 2018 All rights reserved.

\section{Introduction}

Statistical inference for stochastic processes is well developed for diffusion processes. As is wellknown, the maximum likelihood estimate and the bayes estimate have good asymptotic properties under stationarity of the process. However, in addition to asymptotic theory which certainly play a predominant role in statistical theory, sequential estimation has got certain advantages. In the finite dimensional linear SDEs, Novikov [11] (see also Bishwal [1], Liptser and Shiryayev [10]) studied the properties of sequential maximum likelihood estimate (SMLE) of the drift parameter which is the MLE based on observation on a random time interval. He showed that SMLE is better than the ordinary MLE in the sense that the former is unbiased, uniformly normally distributed and efficient (in the sense of having the least variance). His plan is to observe the process until the observed Fisher information exceeds a predetermined level of precision. Many others focused on the extension to other fields, for example, Lee et al. [7], Bo and Yang [2], Kuang and Xie [5] and the references therein.

The present paper is devoted to the sequential maximum likelihood estimation for the following diffusion process:

$$
d X_{t}=\alpha t X_{t} d t+d W_{t} ; X_{0}=x_{0}
$$

Email address: hynuzc1@126.com (Chenglian Zhu)

doi: $10.22436 /$ jnsa.011.06.05

Received: 2017-11-21 Revised: 2018-01-19 Accepted: 2018-03-23 
where $\alpha>0$, and $\left\{W_{t} ; t \geqslant 0\right\}$ is a standard Wiener process. We also assume that usually Lipschitz condition and linear growth condition hold on the coefficient so that there exists a unique solution to (1.1).

Let $P_{\alpha}$ be the probability distribution of $\left\{X_{t} ; t \geqslant 0\right\}$ of the above equation. It is known that, the loglikelihood ratio process of this kind of diffusion model has the representation by Girsanov formula (cf. Bishwal [1]):

$$
\begin{aligned}
l\left(\alpha_{1}, \alpha_{0}\right) & =\left.\log \frac{\mathrm{dP}_{\alpha_{1}}}{\left.\mathrm{dP}\right|_{\alpha_{0}}}\right|_{\mathscr{F}[0, \mathrm{~T}]} \\
& =-\int_{0}^{\mathrm{T}}\left(\alpha_{1}-\alpha_{0}\right) \mathrm{t} X_{\mathrm{t}} \mathrm{d} W_{\mathrm{t}}-\frac{1}{2} \int_{0}^{\mathrm{T}}\left(\alpha_{1}-\alpha_{0}\right)^{2} \mathrm{t}^{2} X_{\mathrm{t}}^{2} \mathrm{dt},
\end{aligned}
$$

where $\mathscr{F}_{[0, \mathrm{~T}]}$ is the $\sigma$-algebra generated by the outcome process realized on $[0, \mathrm{~T}]$. Therefore, the maximum likelihood estimator (MLE) of $\alpha$ based on the observation of $\left\{X_{t} ; 0 \leqslant t \leqslant T\right\}$ under $P_{\alpha}$ is given by

$$
\hat{\alpha}_{T}=\frac{\int_{0}^{T} t X_{t} d X_{t}}{\int_{0}^{T} t^{2} X_{t}^{2} d t} .
$$

In this paper, we propose to use the sequential estimation plan to estimate the unknown parameter $\alpha$ included in the drift term of this kind of diffusion process, respectively.

\section{Sequential maximum likelihood estimation of $\alpha$}

Here and in the sequel, $P_{\alpha}$ be the probability distribution of $\left\{X_{t} ; t \geqslant 0\right\}$. We assume that the process $\left\{X_{t}\right\}$ is observed until the observed Fisher information of the process exceeds a predetermined level of precision $0<H<\infty$, i.e., we observe $\left\{X_{t}\right\}$ over the random time interval $[0, \tau(H)]$, where the random time $\tau(H)$ is a stopping time which is the first time such that the observed information for this kind of the process exceeds $\mathrm{H}$ and is defined as

$$
\tau(H)=\inf \left\{t \geqslant 0 \mid \int_{0}^{t} s^{2} X_{s}^{2} d s \geqslant H\right\},
$$

which is $\mathscr{F}_{\tau(\mathrm{H})}$-measurable (cf. Karatzas and Shreve [4]). Now, we state our main results as follows.

Theorem 2.1. The sequential estimation plan $\left(\tau(\mathrm{H}), \hat{\alpha}_{\tau(\mathrm{H})}\right), 0<\mathrm{H}<\infty$, with the observation time $\tau(\mathrm{H})$ defined as above and the SMLE given by

$$
\hat{\alpha}_{\tau(H)}=\frac{1}{H} \int_{0}^{\tau(H)} t X_{t} d X_{t}
$$

has the following statistical properties:

(i) The sequential plan $\left(\tau(\mathrm{H}), \hat{\alpha}_{\tau(\mathrm{H})}\right)$ defined as above is closed, i.e.,

$$
\mathrm{P}_{\alpha}(\tau(\mathrm{H})<\infty)=1 \text {. }
$$

(ii) The sequential estimator $\hat{\alpha}_{\mathcal{\tau}(\mathrm{H})}$ is unbiased, i.e.,

$$
\mathrm{E}_{\alpha}\left(\hat{\alpha}_{\tau(H)}\right)=\alpha,
$$

where $\mathrm{E}_{\alpha}$ denotes the expectation operator corresponding to the probability measure $\mathrm{P}_{\alpha}$. 
(iii)

$$
\sqrt{\mathrm{H}}\left(\hat{\alpha}_{\tau(H)}-\alpha\right) \stackrel{\mathbb{D}}{=} \mathrm{N}(0,1),
$$

here and in the sequel, $\mathrm{N}(0,1)$ denotes the normal distribution, and $\stackrel{\mathbb{D}}{=}$ denotes the same distribution.

(iv) The sequential estimator is strongly consistent, i.e.,

$$
\hat{\alpha}_{\tau(H)} \stackrel{\text { a.s. }}{\longrightarrow} \alpha,
$$

as $\mathrm{H} \rightarrow \infty$.

Remark 2.2. According to Theorem 2.1, the sequential estimation plan $\left(\tau(H), \hat{\alpha}_{\tau(H)}\right), 0<H<\infty$, has unbiased advantage and the fact that the distribution of the value $\hat{\alpha}_{\tau(H)} \sqrt{H}$ is normal, which makes it possible to construct confidence intervals for $\alpha$.

Proof. Consider an arbitrary stopping time $\tau$ with respect to the filtration $\left\{\mathscr{F}_{t}^{X}\right\}$ generated by the process $X$. By (1.2) and (1.3), it is easy to derive

$$
\hat{\alpha}_{\tau}=\frac{\int_{0}^{\tau} t X_{t} d X_{t}}{\int_{0}^{\tau} t^{2} X_{t}^{2} d t}
$$

Now, let $\tau=\tau(H)$, it has

$$
\hat{\alpha}_{\tau(H)}=\frac{1}{H} \int_{0}^{\tau(H)} t X_{t} d X_{t}
$$

with the fact that

$$
\int_{0}^{\tau(H)} t^{2} X_{t}^{2} d t=H
$$

(i) It is easy to see that for $T>0$

$$
P_{\alpha}(\tau(H) \geqslant T)=P_{\alpha}\left(\int_{0}^{T} t^{2} X_{t}^{2} d t<H\right) .
$$

Thus it suffices to prove

$$
P_{\alpha}\left(\int_{0}^{\infty} t^{2} X_{t}^{2} d t=\infty\right)=1
$$

Apply Itô's formula to the function $x e^{-\frac{\alpha t^{2}}{2}}$, it follows that

$$
X_{t}=e^{\frac{\alpha t^{2}}{2}} \int_{0}^{t} e^{-\frac{\alpha u^{2}}{2}} d W_{u} .
$$

On the other hand, let $\xi_{t}=\int_{0}^{t} e^{-\frac{\alpha u^{2}}{2}} d W_{u}$, since the quadratic variation process

$$
[\xi]_{\mathrm{t}}<\infty .
$$

Thus, $\left\{\xi_{t}\right\}_{t \geqslant 0}$ is a square-integrable $\mathcal{F}_{t}-$ martingale. Moreover, $\xi_{t}$ is a normal random variable with distribution $\mathcal{N}\left(0, \int_{0}^{t} e^{-\alpha u^{2}} d u\right)$. Therefore, by martingale convergence theorem, it follows that

$$
\lim _{t \rightarrow \infty} \xi_{t}=\int_{0}^{\infty} e^{-\alpha u^{2}} d W_{u}
$$


Then, by L'Hospital rule, as $\mathrm{T} \rightarrow \infty$

$$
\frac{\int_{0}^{T} t^{2} X_{t}^{2} d t}{\int_{0}^{T} t^{2} e^{\alpha t^{2}} d t} \rightarrow\left(\int_{0}^{\infty} e^{-\alpha u^{2}} d W_{u}\right)^{2} \text { a.s., }
$$

which is a chi-square random variable. Hence, as $T \rightarrow \infty$

$$
\int_{0}^{T} t^{2} X_{t}^{2} d t \rightarrow \infty \text { a.s.. }
$$

Thus, the desired result is completed.

(ii) Note that

$$
\begin{aligned}
\hat{\alpha}_{\tau(H)} & =\frac{1}{H} \int_{0}^{\tau(H)} t X_{t} d X_{t} \\
& =\frac{1}{H} \frac{1}{H} \int_{0}^{\tau(H)} t X_{t}\left(\alpha t X_{t} d t+d W_{t}\right) \\
& =\frac{1}{H}\left(\alpha \int_{0}^{\tau(H)} t^{2} X_{t}^{2} d t+\int_{0}^{\tau(H)} t X_{t} d W_{t}\right) \\
& =\alpha+\frac{1}{H} \int_{0}^{\tau(H)} t X_{t} d W_{t} .
\end{aligned}
$$

Coupled with the fact that the process $\left\{\int_{0}^{\tau(H)} t X_{t} d W_{t}\right.$ is a Wiener process with variance $H>0$ (see, e.g., Lemma 17.4 in Liptser and Shiryaev [10] or Revuz and Yor [13]). Hence

$$
E_{\alpha}\left(\hat{\alpha}_{\tau(H)}\right)=E_{\alpha}\left(\alpha+\frac{1}{H} \int_{0}^{\tau(H)} t X_{t} d W_{t}\right)=\alpha .
$$

(iii) Observe that

$$
\left(\hat{\alpha}_{\tau(H)}-\alpha\right)^{2}=\frac{1}{H^{2}}\left(\int_{0}^{\tau(H)} \mathrm{t} X_{t} d W_{t}\right)^{2}
$$

and also the stochastic integral $\int_{0}^{\tau(H)} t X_{t} d W_{t}$ is normally distributed with mean 0 and variance $H$. Hence

$$
\sqrt{\mathrm{H}}\left(\hat{\alpha}_{\tau(H)}-\alpha\right)=\frac{1}{\sqrt{\mathrm{H}}} \int_{0}^{\tau(\mathrm{H})} \mathrm{t} X_{\mathrm{t}} \mathrm{d} W_{\mathrm{t}} \stackrel{\mathbb{D}}{=} \frac{1}{\sqrt{\mathrm{H}}} \mathrm{N}(0, \mathrm{H}) \stackrel{\mathbb{D}}{=} \mathrm{N}(0,1) .
$$

Then, the desired result follows.

(iv) Due to

$$
\int_{0}^{\tau(H)} t^{2} X_{t}^{2} d t=H
$$

and

$$
\frac{1}{\sqrt{\mathrm{H}}} \int_{0}^{\tau(\mathrm{H})} \mathrm{t} X_{\mathrm{t}} \mathrm{dW} \mathrm{t} \rightarrow 0 \text { a.s. }
$$

as $H \rightarrow \infty$, which follows from the fact that $\int_{0}^{\tau(H)} t X_{t} d W_{t} \stackrel{D}{=} N(0, H)$, the desired result follows immediately. 
Next, we show that the proposed sequential estimation plan $\left(\tau(H), \hat{\alpha}_{\tau(H)}\right)$ for estimating parameter $\alpha$ of the diffusion process $\left\{X_{t}\right\}$ defined by (1.1) is efficient among all unbiased estimation plans in the mean squared error sense (MSE). In fact, we will prove an analog of the Cramer-Rao lower bound for arbitrary unbiased estimation plans.

Theorem 2.3. Let the sequential plan $\left(\tau, \hat{\alpha}_{\tau}\right)$ be an arbitrary unbiased estimation plan for the diffusion process $\left\{X_{t}\right\}$ defined by (1.1). Suppose that $0<\mathrm{E}_{\alpha}\left(\int_{0}^{\tau} \mathrm{t}^{2} \mathrm{X}_{\mathrm{t}}^{2} \mathrm{dt}\right)<\infty$. Then

$$
\operatorname{Var}_{\alpha}\left(\hat{\alpha}_{\tau}\right)=E_{\alpha}\left(\hat{\alpha}_{\tau}-\alpha\right)^{2} \geqslant \frac{1}{E_{\alpha}\left(\int_{0}^{\tau} t^{2} X_{t}^{2} d t\right)}
$$

Remark 2.4. A sequential plan $\left(\tau, \hat{\alpha}_{\tau}\right)$ is said to be efficient in the MSE sense if for which the equality holds true. Observe that $E_{\alpha}\left(\hat{\alpha}_{\tau(H)}-\alpha\right)^{2}=\frac{1}{H}$ and the definition of $\tau(H)$, the sequential plan $\left(\tau(H), \hat{\alpha}_{\tau(H)}\right)$ is efficient in the MSE sense.

Remark 2.5. Based on continuous observations, the maximum likelihood estimator $\hat{\alpha}_{T}^{M L E}$ for an unknown drift parameter $\alpha$ of this kind of diffusion process is derived, and strong consistency and asymptotic normality of this estimation is established (cf Kutoyants [6] and Prakasa [12]). However, the classical Cramer-Rao lower bound (cf Liptser [10]) for this MLE may not be attained. In fact, as defined in (1.3), the estimator $\hat{\alpha}_{T}^{M L E}$ is naturally defined as

$$
\hat{\alpha}_{\mathrm{T}}:=\arg \sup _{\alpha \in \Theta} l(\alpha), l(\alpha)=l\left(\alpha, \alpha_{0}\right) .
$$

Then, we can derive the maximum likelihood estimator (MLE) of the parameter $\alpha$ by solving the likelihood equation $l^{\prime}(\alpha)=0$. On the other hand, it follows that

$$
l^{\prime}(\alpha)=-\int_{0}^{T} t X_{t} d W_{t}
$$

and

$$
l^{\prime \prime}(\alpha)=\int_{0}^{T} t^{2} X_{t}^{2} d t
$$

Thus, in view of Taylor's expansion, it follows that

$$
l^{\prime}(\alpha)=l^{\prime}\left(\hat{\alpha}_{T}^{M L E}\right)+\left(\alpha-\hat{\alpha}_{T}^{M L E}\right) l^{\prime \prime}\left(\xi_{T}\right),
$$

where $\left|\xi_{T}\right| \leqslant 1$. Then

$$
b(\alpha)=E_{\alpha}\left(\hat{\alpha}_{T}^{M L E}-\alpha\right)=E_{\alpha}\left(\frac{-l^{\prime}(\alpha)}{\int_{0}^{T} t^{2} X_{t}^{2} d t}\right),
$$

which, together with (2.1), implies that

$$
\mathrm{b}^{\prime}(\alpha)=\mathrm{E}_{\alpha}\left(\frac{\mathrm{l}^{\prime \prime}(\alpha)}{\int_{0}^{\mathrm{T}} \mathrm{t}^{2} X_{\mathrm{t}}^{2} d t}\right)=-1
$$

Therefore, it is obvious to observe that the equality of Theorem 7.22 in Liptser [9] does not follow. Hence the maximum likelihood estimator $\hat{\alpha}_{\mathrm{T}}^{\mathrm{MLE}}$ may not attain this class of lower bound. 
The proof of Theorem 2.3. In view of the Radon-Nikodym derivative formula in (1.2) and Girsanov's Theorem, we have

$$
\begin{aligned}
1=\frac{\partial}{\partial \alpha} E_{\alpha}\left(\hat{\alpha}_{\tau}\right) & =\frac{\partial}{\partial \alpha} E_{\alpha_{0}}\left(\hat{\alpha}_{\tau} e^{\left.\left.l\left(\alpha, \alpha_{0}\right)\right|_{T=\tau}\right)}\right. \\
& =E_{\alpha_{0}}\left(\left.\hat{\alpha}_{\tau} e^{\left.l\left(\alpha, \alpha_{0}\right)\right|_{T=\tau}} l_{\alpha}^{\prime}\left(\alpha, \alpha_{0}\right)\right|_{T=\tau}\right) \\
& =E_{\alpha_{0}}\left(\hat{\alpha}_{\tau} e^{\left.l\left(\alpha, \alpha_{0}\right)\right|_{T=\tau}}\left\{\alpha \int_{0}^{T} t^{2} X_{t}^{2} d t-\int_{0}^{T} t X_{t} d X_{t}\right\}\right) \\
& =E_{\alpha}\left(\hat{\alpha}_{\tau}\left\{\alpha \int_{0}^{T} t^{2} X_{t}^{2} d t-\int_{0}^{T} t X_{t} d X_{t}\right\}\right) .
\end{aligned}
$$

Also since

$$
E_{\alpha}\left(\alpha \int_{0}^{T} t^{2} X_{t}^{2} d t-\int_{0}^{T} t X_{t} d X_{t}\right)=E_{\alpha}\left(\alpha \int_{0}^{T} t^{2} X_{t}^{2} d t-\int_{0}^{T} t X_{t}\left(\alpha t X_{t} d t+d W_{t}\right)\right)=0,
$$

it follows that

$$
E_{\alpha}\left(\left(\hat{\alpha}_{\tau}-\alpha\right)\left\{\alpha \int_{0}^{T} t^{2} X_{t}^{2} d t-\int_{0}^{T} t X_{t} d X_{t}\right\}\right)=1
$$

Then, by Cauchy-Schwarz inequality, we have

$$
\begin{aligned}
1 & \leqslant E_{\alpha}\left(\hat{\alpha}_{\tau}-\alpha\right)^{2} E_{\alpha}\left(\left\{\alpha \int_{0}^{T} t^{2} X_{t}^{2} d t-\int_{0}^{T} t X_{t} d X_{t}\right\}\right)^{2} \\
& =E_{\alpha}\left(\hat{\alpha}_{\tau}-\alpha\right)^{2} E_{\alpha}\left(\int_{0}^{\tau} t X_{t} d W_{t}\right)^{2} \\
& =E_{\alpha}\left(\hat{\alpha}_{\tau}-\alpha\right)^{2} E_{\alpha}\left(\int_{0}^{\tau} t^{2} X_{t}^{2} d t\right) .
\end{aligned}
$$

Therefore, the desired result follows from the assumption of our Theorem.

\section{Concluding remarks}

Generally speaking, the ordinary MLE for an unknown drift parameter of the diffusion process can be established some statistical properties, including strong consistency and asymptotic normality. However, the MLE may be a biased estimator and the classical Cramer-Rao lower bound may not be attained for the MLE (cf. Prakasa [12]; Liptser [10] ). To overcome such limitations, in this paper, we propose to use the sequential estimation plan for this kind of diffusion process and verify that the proposed plan is significantly helpful both in asymptotic and non-asymptotic short time observation.

More precisely, in contrast to the MLE, the proposed sequential maximum likelihood estimator (SMLE) is unbiased, exactly normally distributed (on the finite time observation), and its mean squared error(MSE) has an explicit, simple expression that does not depend on the parameter to be estimated. The SMLE is uniformly normally distributed over the entire parameter space which is the real line. Such results would be of ample use in applications to several areas such as engineering, financial and biological modeling where unknown parameter estimation is based on relatively shorter time observation, which commonly arises in practical situations. Furthermore, an analog of the Cramer-Rao lower bound is proved and the SMLE is shown to be efficient among all unbiased estimation plans in the mean squared error sense.

On the other hand, some future work may investigate some other estimators for the other diffusions. See, for example, Lee et al. [7] proposed a sequential maximum likelihood estimation of the unknown drift of the reflected Ornstein-Uhlenbeck process without jumps; some others are concerned with the problem of statistical parameter estimation for reflected fractional Brownian motion (cf. Hu and Lee [3]; Lee and Song [8]). 


\section{Acknowledgment}

The author also acknowledges partial research support from National Natural Science Foundation of China (Grant Nos. 11326174 and 11401245), Natural Science Foundation of Jiangsu Province (Grant No. BK20130412), Natural Science Research Project of Ordinary Universities in Jiangsu Province (Grant No. 12KJB110003), Qing Lan project of Jiangsu Province (2016).

\section{References}

[1] J. P. N. Bishwal, Parameter estimation in stochastic differential equations, Lecture Notes in Mathematics, Springer, New York, (2008). 1, 1

[2] L. Bo, X. Yang, Sequential maximum likelihood estimation for reflected generalized Ornstein-Uhlenbeck processes, Statist. Probab. Lett., 82 (2012), 1374-1382. 1

[3] Y. Hu, C. Lee, Parameter estimation for a reflected fractional Brownian motion based on its local time, J. Appl. Probab., 50 (2013), 592-597. 3

[4] I. Karatzas, S. E. Shreve, Brownian motion and stochastic calculus, Springer-Verlag, New York, (1991). 2

[5] N. Kuang, H. Xie, Sequential maximum likelihood estimation for the hyperbolic diffusion process, Methodol. Comput. Appl. Probab., 17 (2015), 373-381. 1

[6] Y. A. Kutoyants, Statistical inference for ergodic diffusion processes, Springer-Verlag, London, (2004). 2.5

[7] C. Lee, J. P. N. Bishwal, M. H. Lee, Sequential maximum likelihood estimation for reflected Ornstein-Uhlenbeck processes, J. Statist. Plann. Inference, 142 (2012), 1234-1242. 1, 3

[8] C. Lee, J. Song, On drift parameter estimation for reflected fractional Ornstein-Uhlenbeck processes, Stochastics, 88 (2016), 751-778. 3

[9] R. S. Liptser, A. N. Shiryayev, Statistics of random processes I, General Theory, Springer-Verlag, New York, (1977). 2.5

[10] R. S. Liptser, A. N. Shiryayev, Statistics of random processes II, Applications, Springer-Verlag, New York, (1977). 1, 2, $2.5,3$

[11] A. A. Novikov, Sequential estimation of the parameters of diffusion processes (Russian), Mat. Zametki, 12 (1972), 627638. 1

[12] B. L. S. Prakasa Rao, Statistical inference for diffusion type processes, Oxford University Press, New York, (1999). 2.5, 3

[13] D. Revuz, M. Yor, Continuous martingales and brownian motion, Third edition, Springer-Verlag, Berlin, (1999). 2 